\title{
Proton Transfer Reaction in Water: Hydronium Ion Formation
}

In Sang Leea, Sitansu Sekhar Nanda ${ }^{\mathrm{b}, *}$

\section{ART I CLE I N F O}

Received: 28 June 2019

Revised: 07 August 2019

Accepted: 31 August 2019

Available online: 03 September 2019

\section{K E Y W O R D S}

Raman spectroscopy

Hydronium

Water

Infrared spectroscopy

\section{A B S T R A C T}

The current ongoing scientific debate deals with accumulation of hydronium ions $\left(\mathrm{H}_{3} \mathrm{O}^{+}\right)$on water surface. Elevated interfacial concentration measured by using Raman spectroscopy. A strong surface affinity of $\mathrm{H}_{3} \mathrm{O}^{+}$indicated by Raman spectroscopy under similar conditions. Ion adsorption phenomena, $\mathrm{H}_{3} \mathrm{O}^{+}$ formation and its structural activity emphasized in our study. Asymmetric water ion adsorption clearly observed in our research.

\section{GRA P H I C A L A B S T RACT}
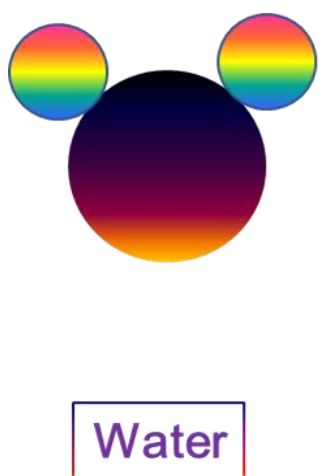
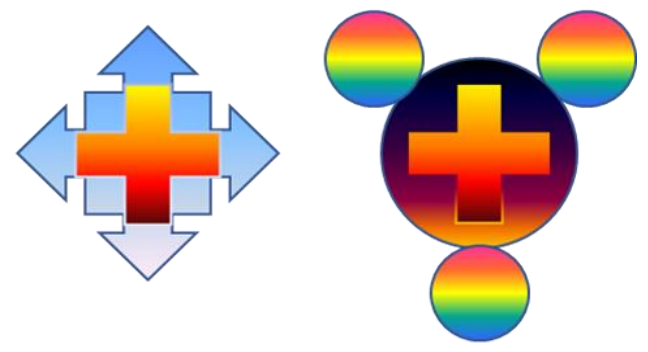

Hydronium

\footnotetext{
* Corresponding author's E-mail address: nandasitansusekhar@gmail.com
} 


\section{Introduction}

In nature, interfacial charge formation is known as a ubiquitous phenomenon. Fundamental mechanism of interfacial charge formation related with its identification. Researchers have been attracted for charge formation development of appropriate models. In our research, we studied charge formation at the molecular level by using computational and analytical methods.

Dissimilar processes have been involved in interface charge. It is caused by substitution, desorption or adsorption of lattice ions. In case of solid surfaces, ionization of functional groups was responsible for interface charge [1]. This phenomenon also observed in case of aqueous solutions [2-9].

\section{Experimental}

Faraday's law utilized for synthesis of $\mathrm{H}_{3} \mathrm{O}^{+}$. Two electrodes or plates were supplied, made up with stainless steel, leads to hydrogen and oxygen ions dissociation. Negatively charged electrode (cathode) deposited hydrogen while positively charged electrode oxygen (anode) deposited oxygen. In calculation of faraday efficiency, hydrogen generation is twice than that of oxygen in moles.

\section{Raman measurements}

A Renishaw micro-Raman system was used for the acquisition of Raman spectra with a grating of 1800/500 g/mm-1. He-Ne $633 \mathrm{~nm}$ laser was utilized for the source of excitation on the sample with a power of $70 \mu \mathrm{W}$. A working distance of $8 \mathrm{~mm}$, a numerical aperture of 0.55 and a 10x objective (Olympus) lens were employed for recording Raman spectra with 2$10 \mathrm{~s}$ acquisition time. A scanning area of $30 \mu \mathrm{m} \times$ $30 \mu \mathrm{m}$ was applied to avoid laser damage to water.

\section{Results and discussions}

As mentioned above, we studied the electronic properties, stability and reactivity of Zonisamide in our previous article. We showed the B3LYP/6-311++G(d,p) basis set of theory is the best computational method to study this compound and its analogues [19]. So, this computational method will be used to optimize our new designed compounds. Scheme 1 shows the molecular structure of the novel designed molecules.

Water has been considered as most essential component in living world. Some unusual and significant properties are involved in water such as its physical properties, chemical properties, solvent potency, hydrogen bonds forming ability and amphoteric natures [10]. Biologists proved that life's molecular components development related with water. This helps to our research that $\mathrm{H}_{3} \mathrm{O}^{+}$will serve as a better option for the surface of water. In neighboring water molecules, three hydrogen donor bonds formed. Oxygen atom kept the positive charge and became hydrophobic. From this whole process, $\mathrm{H}_{3} \mathrm{O}^{+}$ proved as an amphiphilic nature [10]. Infrared data provided in Figure 2, and it is having 95.62\% similarity with sodium hypochlorite. Sodium hypochlorite is widely known as bleaching agent.

In this way, presence of oxygen atom at outermost, $\mathrm{H}_{3} \mathrm{O}^{+}$surface accumulation encouraged by reduced hydrogen-bond capacity [11].

Figure 1. Schematic illustration of $\mathrm{H}_{3} \mathrm{O}^{+}$

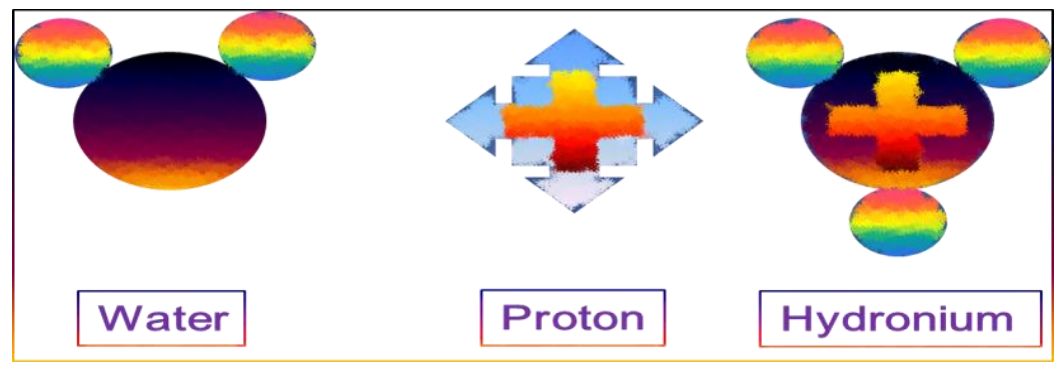


Figure 2. The above figure depicted infrared spectrum of water and $\mathrm{H}_{3} \mathrm{O}^{+}$. It clearly indicated that structural dissimilarity found in both study

Figure 3. The above Figure illustrates Raman spectrum of water and $\mathrm{H}_{3} \mathrm{O}^{+}$. There was a structural dissimilarity observed. This was proved by using computational methods
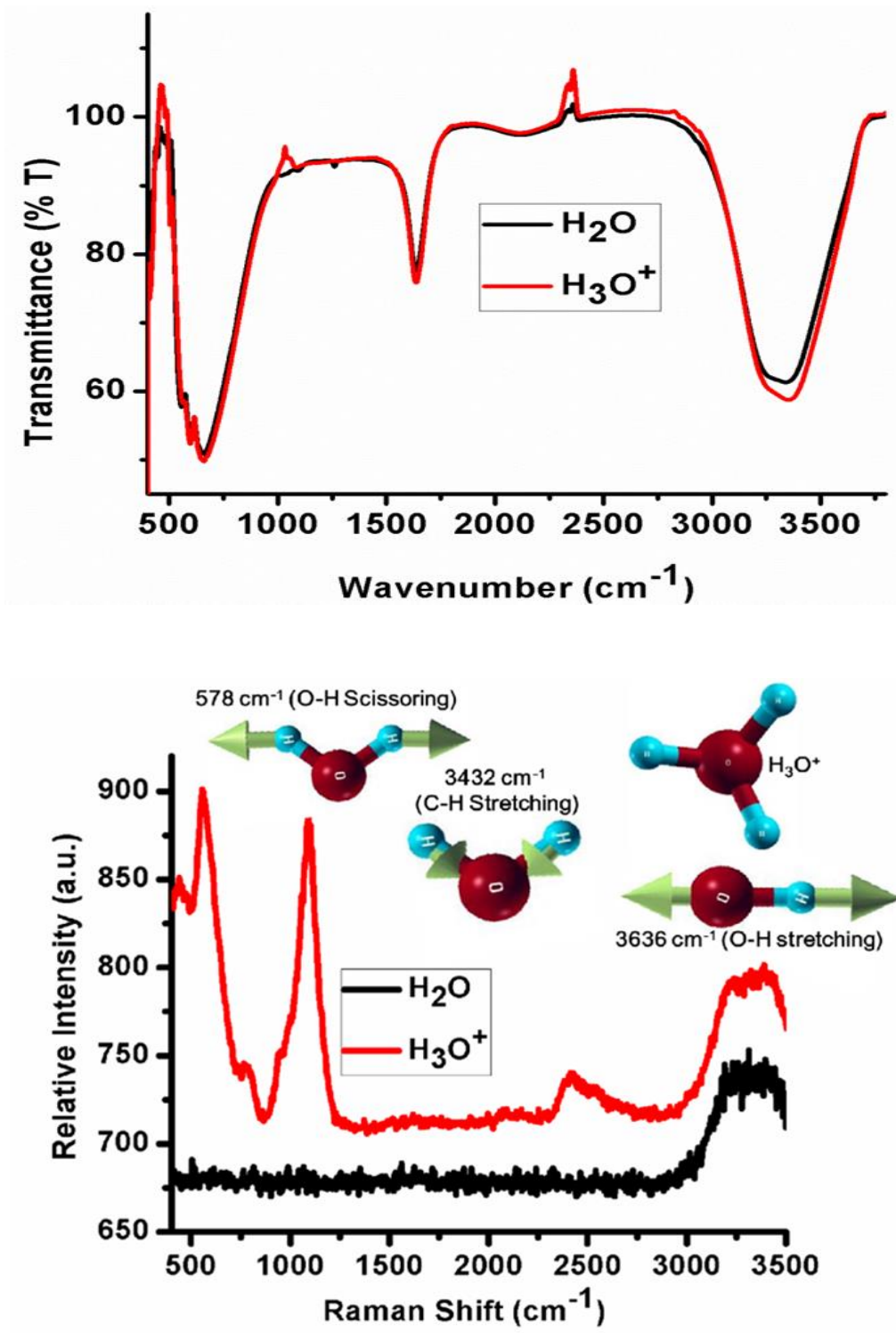

Figure 4. Raman spectrum of $\mathrm{H}_{3} \mathrm{O}^{+}$in different time intervals (30 min, $1 \mathrm{hr}, 2 \mathrm{hr}$ and 4 hr). It was mostly similar after $2 \mathrm{hr}$ study

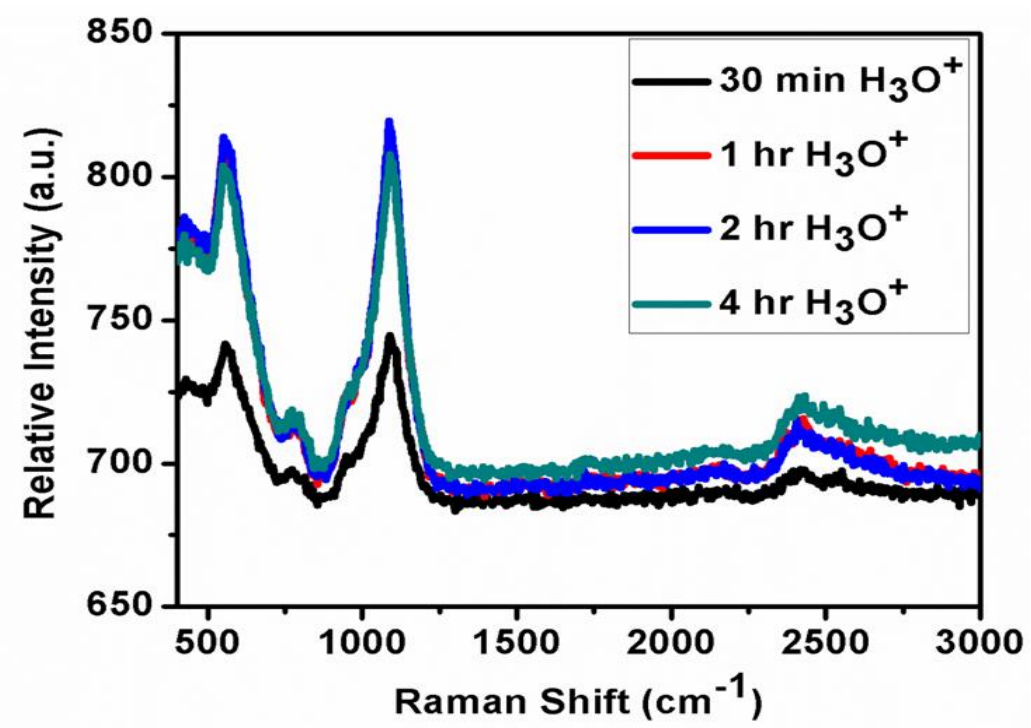


Hydrogen-bond capacity have important role in biomolecular hydration at hydrophobic surfaces. We expected that dissociation of protonable residues shifted near to hydrophobic patches. After this, stabilization of hydrophobic species occurred and $\mathrm{H}_{3} \mathrm{O}^{+}$showed surfactant behavior [12]. O-H scissoring, $\mathrm{C}-\mathrm{H}$ stretching and $\mathrm{O}-\mathrm{H}$ stretching were demonstrated in Figure 3.

To better understand the electronic processes taking place at the water interface, an empirical model was developed through firstprinciples calculations. We used density functional theory (DFT) (implemented in the QUANTUM ESPRESSO simulation package) [13] to simulate the charge transfer mechanism. Generalized gradient approximation (GGA) was used to exchange the correlation energy of electrons [14] and ultra-soft pseudopotentials [15] to represent the interaction between ionic cores and valence electrons. Plane wave basis was used with an energy cut off of 240 Ry. Suitable grid mesh was used for Brillouin zone (BZ) sample integration [16].

\section{Conclusion}

Ultimately, proton transport facilitated by $\mathrm{H}_{3} \mathrm{O}^{+}$ion was derived from water molecules. This process continued from one molecule to another molecule. Diffusion of specific proton does not occurred throughout the water medium. Cooperative transfer of protons demonstrated successive molecules.

\section{Acknowledgments}

We are thankful to Mr. Choi and Mr. Oh for their contribution.

\section{Disclosure statement}

No potential conflict of interest was reported by the authors.

\section{References}

[1] A.V. Delgado, F.J. Arroyo, Electrokinetic phenomena and their experimental determination: an overview. In Interfacial electrokinetics and electrophoresis, CRC Press, 2001, pp. 21-74.

[2] C. Dicke, G. Hähner, J. Am. Chem. Soc., 2002, 124, 12619-12625.

[3] A. Hozumi, H. Sugimura, Y. Yokogawa, T. Kameyama, O. Takai, Colloids Surf A, 2001, 182, 257-261.

[4] A. Quinn, R. Sedev, J. Ralston, J. Phys. Chem. B, 2003, 107, 1163-1169.

[5] R. Schweiss, P.B. Welzel, C. Werner, W. Knoll, Langmuir, 2001, 17, 4304-4311.

[6] A. Härtl, J.A. Garrido, S. Nowy, R. Zimmermann, C. Werner, D. Horinek, R. Netz, M. Stutzmann, J. Am. Chem. Soc., 2007, 129, 1287-1292.

[7] Y.H.M. Chan, R. Schweiss, C. Werner, M. Grunze, Langmuir, 2003, 19, 7380-7385.

[8] J.K. Beattie, Lab Chip, 2006, 6, 1409-1411.

[9] R. Zimmermann, S. Dukhin, C. Werner, J. Phys. Chem. B, 2001, 105, 8544-8549.

[10] M.K. Petersen, S.S. Iyengar, T.J. Day, G.A. Voth, J. Phys. Chem. B, 2004, 108, 1480414806.

[11]. S.S. Iyengar, T.J.F. Day, G.A. Voth, Int. J. Mass Spectrom., 2005, 241, 197-204.

[12] C.J.T. De Grotthuss, Biochim. Biophys. Acta (BBA)-Bioenerget, 2006, 1757, 871-875.

[13] J.P. Perdew, K. Burke, M. Ernzerhof, Phys. Rev. Lett., 1996, 77, 3865-3868.

[14] D. Vanderbilt, Phys. Rev. B: Condens. Matter Mater. Phys., 1990, 41, 7892.

[15] M. Methfessel, A.T. Paxton, Phys, Rev. B: Condens. Matter Mater. Phys., 1989, 40, 3616-3621.

[16] H.J. Monkhorst, J.D. Pack, Phys. Rev. B: Solid State, 1976, 13, 5188.

How to cite this manuscript: In Sang Lee, Sitansu Sekhar Nanda, Proton Transfer Reaction in Water: Hydronium Ion Formation, Adv. J. Chem. A, 2020, 3(3), 255-258. 